\title{
SIMON STRICK
}

\section{ALT-RIGHT-AFFEKTE}

\section{Provokationen und Online-Taktiken}

«All the messages are emotional.»

LAURENT BERLANT, Trump, or Political Emotions

Für den englischen Begriff prank gibt es keine angemessene Übersetzung. Ein $<$ Streich $>$ im Deutschen trägt leicht altmodische Konnotationen. Er assoziiert Kinder, eine vielleicht kleinstädtische Umgebung, ein unschuldiges Verwirrspiel mit den Routinen der Ewachsenenwelt ohne ernste Konsequenz. <Klingelstreich〉 und 〈Bubenstreich〉 gibt das Online-Wörterbuch leo.org als Äquivalente mit altbackener Geschlechtsspezifik aus. Die Begriffe klingen nach kleiner Welt, in der Jungs eben tun, was Jungs so tun. Prank in der Internetsphäre verbindet diese Intimität mit globaler Reichweite und extremer Sichtbarkeit, denn das Internet ist «just a world passing around notes in a classroom», wie TVTalker Jon Stewart einmal sagte.

Im Folgenden vergleiche ich zwei pranks aus dem derzeitigen Kulturkampf, der von der US-amerikanischen Alt-Right lanciert wird. Beide zirkulierten im vergangenen Jahr und erzielten aus dem virtuellen Raum heraus emotional und politisch folgenreiche Effekte.

Zur Begriffsklärung: Alt-Right (alternative right), geprägt vom weißen Suprematisten Richard Spencer, bezeichnet ein informelles Netzwerk aus Vertreter_ innen von Ethnonationalismus, Demokratiefeindlichkeit, Antifeminismus und Rassismus. ${ }^{1}$ Die Alt-Right ist von den politischen Feldern, die als $<$ Rechtspopulismus > oder <klassischer〉 Neofaschismus kanonisiert sind, weder personell noch ideologisch sauber zu trennen: Die unscharfe Verhandlung dieser Ideologeme ist Charakteristikum der neuen Rechten. Alt-Right bezeichnet im amerikanischen Kontext primär eine onlinebasierte Bewegung, die dissidentisch zur etablierten Politik und Parteienlandschaft steht, links wie rechts. Die Alt-Right geriert sich als intellektuelle, transgressive und mediensteuernde Avantgarde zur Reinstallation weißer, männlicher Vorherrschaft. Ihre Verfahrensweise, so meine These, ist die Produktion von Affekt und die Arbeit am Affekt.

1 Vgl. George Hawley: Making Sense of the Alt-Right, New York 2017. 
2 Vgl. Whitney Phillips, Ryan Milner: The Ambivalent Internet: Mischief, Oddity, and Antagonism Online, Cambridge 2017.

3 Vgl. Nancy S. Love: Back to the Future: Trendy Fascism, the Trump Effect, and the Alt-Right, in: New Political Science, Vol. 39, Nr. 2, 2017 , 263-268.

4 N. N.: The 100 Most Influential People, in: TIME Magazine, dort datiert 21.4.2016, time.com/ collection/2016-time-100, gesehen am 28.6.2018.

5 Vgl. Gabriele Dietze, Simon Strick: Aufstand der Betamännchen, in: Gender Blog, Zeitschrift für Medienwissenschaft, dort datiert 18.12.2017, zfmedienwissenschaft.de/online/blog/deraufstand-der-betamännchen, gesehen am 28.6.2018.

6 PewDiePies geschätzte Einnahmen pro Video liegen bei 21.000 USD, das Jahreseinkommen bei 11 Millionen USD. Sponsoren sind z. B. MTV, der Computerhersteller Razer und Disney. Vgl. Bill Roberts: How much does PewDiePie make? Full YouTube Earnings Report!, in: Vloggergear, dort datiert 8.11.2017, vloggergear.com/howmuch-does-pewdiepie-make, gesehen am 28.6.2018.

7 Ausnahme: Marcus Maloney, Steven Roberts, Alexandra Caruso: ‘Mmm ... I love it, bro!?: Performances of masculinity in YouTube gaming, in: New Media \& Society, Vol. 20, Nr. 5, 2018, 1697-1714.

8 PewDiePies originaler Upload wurde infolge der Kontroverse von dessen Kanal entfernt, findet sich aber in zahlreichen Re-Uploads auf YouTube und anderen Portalen wieder, youtube.com/watch?v=jGPq $\mathrm{D}_{3} \mathrm{Pn} \mid 20$ \& frags $=\mathrm{pl} \% 2 \mathrm{Cwn}$, gesehen am 10.7.2018. Kjellberg selbst hat mehrere Entschuldigungs- und Relativierungsvideos hochgeladen, die das Video zitieren und somit teilweise öffentlich machen.

9 Funny Guys: Selbstbeschreibung, in: Fiverr, fiverr.com/funnyguys, gesehen am 28.6.2018.
Der Vergleich zweier pranks - des YouTubers PewDiePie und einer online organisierten Posteraktion - soll aufzeigen, dass die Sprach-, Aufmerksamkeits- und Netzwerkspiele innerhalb sozialer Medien nicht nur <ambivalent> und vieldeutig sind, wie Milner und Phillips in ihrer Studie zu Antagonismen im Internet ${ }^{2}$ ausführen. Vielmehr wird deutlich, dass konventionalisierte Verhaltensweisen und Affekte der Social Media - wie Trolling, Ironie, humoristischer Kommentar, metamediale Produktion - mittlerweile im Flächeneffekt dorthin tendieren, wo wir gemeinhin <rechts $>$ oder $<$ rechtspopulistisch $>$ situieren. Der unternehmerische Aspekt ist hier nicht zu unterschätzen: Es ist für <politisch neutrale> YouTuber wie PewDiePie äußerst lukrativ, Provokationen zu produzieren und affektiv durchzuarbeiten, die jenen nationalistischen und rassistischen Positionen nahestehen, die im zweiten Beispiel urhebend sind. Als Affektarbeiter in der nervösen Aufmerksamkeitsökonomie der Plattform kommen ihm und der Alt-Right die Aufregung um Rassismus zur Produktion authentischer Gefühle gerade recht - rechter Populismus und «flirting with fascism $\gg^{3}$ sind emotional, diskursiv und finanziell sinnvoll. Die Abonnent_ innenzahlen und view counts steigen nicht trotz, sondern wegen der ausgelösten Kontroversen unbeirrt.

\section{PewDiePie}

Der $<$ Streich〉, den PewDiePie am I I. Januar 2017 als Video veröffentlichte, erreichte ein Millionenpublikum. PewDiePie, bürgerlich Felix Kjellberg, ist seit Dezember $20{ }_{3}$ fast ununterbrochen der populärste YouTube-Kanal (derzeit 63 Millionen Abonnent_innen). Für die Videoplattform sind seine täglichen Vlogs und Videos so zentral wie der <Tatort> für die deutsche Fernsehlandschaft. Laut Time Magazine $e^{4}$ ist Kjellberg einer der roo einflussreichsten Menschen der Welt, und er kann als Protagonist jenes Online-Milieus juveniler Männlichkeiten bezeichnet werden, die Gabriele Dietze und ich an anderer Stelle die <Betamännchen $>$ genannt haben. ${ }^{5}$ Trotz seiner Reichweite und Popularitä ${ }^{6}{ }^{6}$ ist der 29 -jährige Schwede akademisch fast nicht rezipiert. ${ }^{7}$

Das fragliche Video $^{8}$ zeigt PewDiePie in seinem Aufnahmestudio, als er die Internetplattform Fiverr entdeckt, um sie 〈humoristisch〉 zu rezensieren. Das israelische Unternehmen der sogenannten Gig Economy bietet eine Plattform, ähnlich wie Uber oder Amazons Mechanical Turk, auf der Nutzer_innen die Dienstleistungen von Menschen auf der ganzen Welt erwerben können, beginnend beim Gebot von fünf Dollar, einem fiver.

Kjellberg entscheidet sich dafür, dem indischen Anbieter Funny Guys ein Angebot zu machen, dessen Selbstbeschreibung lautet: «We are funny guys. Me and my cousins have good skills in making videos. We make funny videos, Happy birthday wishes, promoting your business/product, Website advertising and any promotional videos. Your satisfaction is our success. ${ }^{9}$ Kjellberg gibt den Auftrag, dass ein Schild mit der Aufschrift «Death To All Jews - Subscribe 
to Keemstar» hochgehalten werden soll. Nach der live erfolgten Auftragsabgabe surft er weiter durch die Angebote und bewertet schließlich die Ergebnisse. Der Schnitt verbirgt, dass mehrere Tage zwischen Auftrag und Lieferung vergangen sind - das Video präsentiert den gesamten Vorgang in einer I 5-minütigen Collage. Zuletzt sehen wir einen reaction shot: eine Einstellung, in der das gelieferte Video der Funny Guys unten links als Insert läuft, während wir im größeren Rest des Bildes Kjellbergs vermeintliche Echtzeit-Reaktion sehen. Der Funny-Guys-Film zeigt zwei Männer mit bloßem Oberkörper und kurzen Hosen vor einem Wald. Sie haben Christbaumdekorationen um die Hälse gelegt. Die Männer lachen zunächst längere Zeit und streiten sich dann um eine Papierrolle. Der linke gewinnt die Auseinandersetzung, entrollt das Papier mit der Aufschrift «DEATH TO ALL JEWS» und spricht die Worte: «Subscribe to Keemstar». ${ }^{10}$ Beide Männer lachen und tanzen.

Im simultanen reaction shot sehen wir einen zunächst skeptischen PewDiePie ( «I paid for this?!»), der das unbeholfene Tanzen und Lachen der Funny Guys nicht überzeugend findet. Als das Schriftband entrollt wird, hält er sich <im Schock > die Hand vor den Mund. Seine Augen sind weit aufgerissen. Als das Fiverr-Video zu Ende ist, ringt Kjellberg nach Worten, ein Schnitt zeigt sein Gesicht nun größer. Sein Blick ist auf den Monitor vor ihm gerichtet, nur kurz schaut er in die Kamera. Er sagt: «I am sorry. I didn't think they would actually do it. I feel partially responsible.» Mit einem weiteren jumpcut fährt die Kamera wieder heraus und ein erstes, befreiendes Lachen ist hörbar. Kjellberg fährt fort und bewertet auf der Fiverr-Homepage das Ergebnis mit den Worten: «I mean I've got to give them five stars for an outstanding experience because at least they did what I asked.» Zum Abschluss des Videos kommt er zum emotionalen Fazit des pranks und nimmt eine Art Live-Bewertung der eigenen Aktion und der sich anschließenden Gefühle vor:

I don't feel good. I don't feel too proud of this, I'm not gonna lie. I'm not antisimetic [sic], or whatever it's called, okay so don't get the wrong idea. It was a funny meme, and I didn't think it would work, okay. I swear I love jews, I love'em.

Der Studienabbrecher Kjellberg hat eine Karriere und ein Geschäftsimperium aus exaggerated emoting gebaut, dem übertriebenen Ausstellen exaltierter Emotion. PewDiePies Brand ist eine Art Klassenclown des Internets, der juvenile Reaktionsmuster überdreht und übertrieben durchspielt. Die pranks, die sich innerhalb der Genres und Routinen virtueller Hypermedialität fabrizieren lassen, gehören seit Jahren zu Kjellbergs Unterhaltungsformat. Auf seinem Kanal finden sich viele ähnliche Videobeiträge, in denen er die möglichen Verdrehungen heutiger Aufmerksamkeitsökonomien ausstellt und das Internet zum Transgressionsinstrument umwidmet. Olga Goriunova hat solche Online-Phänomene hilfreich als «New Media Idiocy» betitelt. ${ }^{11}$

Kjellberg wurde in der Folge von YouTube demonetarisiert, ${ }^{12}$ von seiner Fangemeinde unter dem Hashtag \#FreePewDiePie verteidigt und von
10 Keemstar ist ein Konkurrenzkanal von PewDiePie und bekannt für seine häufige Verwendung von Beleidigungen bis hin zu hate speech. Vgl. dazu Synge: Keemstar, in: knowyourmeme, dort datiert 21.3.2016, knowyourmeme.com/ memes/people/keemstar, gesehen am 28.6.2018.

11 Vgl. Olga Goriunova: New media idiocy, in: Convergence, Vol. 19, Nr. 2, 2013, 223-235.

12 Demonetarisierung bedeutet, dass YouTube bestimmte Kanäle, Produzent_innen oder auch einzelne Videos von möglichen Einnahmen durch Werbung ausschließt. YouTube behält sich die Maßnahme für «nicht-werbefreundliche Inhalte» vor. Für die hier diskutierten YouTube-Entrepreneurs bedeutet dies empfindliche Umsatzeinbußen, auch wenn die meisten von ihnen über Zweit- und Drittfinanzierungen (z. B. Patreon.com) verfügen. Demonetarisierung wird seit der Einführung als informelle ‘Zensurs seitens der Plattform diskutiert. Vgl. YouTube: Richtlinien für werbefreundliche Inhalte, support.google.com/youtube/ answer $/ 6162278$ ?vid $=0-1220299$ 005482-1529840735822, gesehen am 28.6.2018; vgl. Rolfe Winkler, Jack Nicas, Ben Fritz: Disney Severs Ties with YouTube Star PewDiePie after Antisemitic Posts, in: Wall Street Journal, 14.2.2017. 

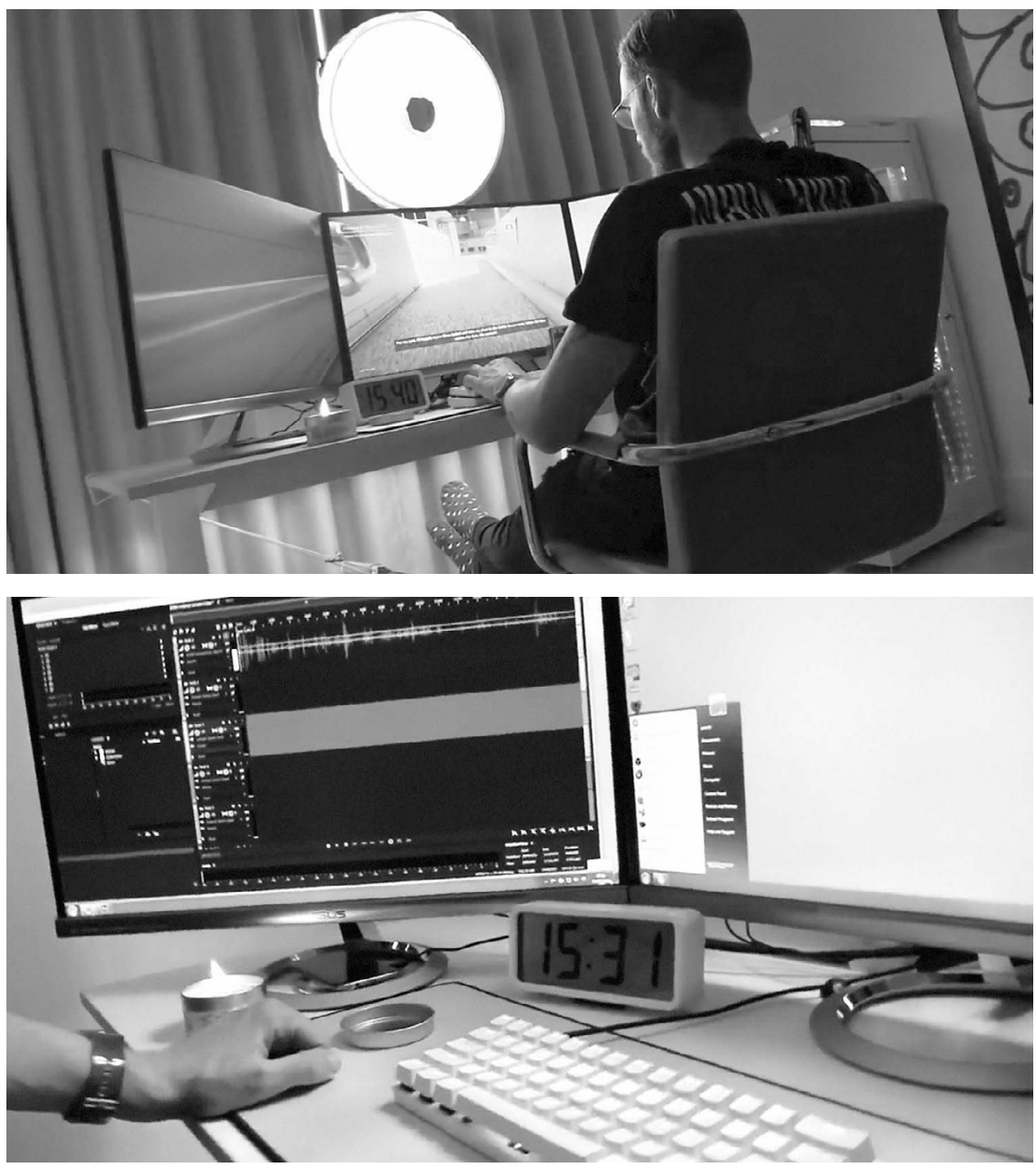
neofaschistischen Portalen wie The Daily Stormer als Held der Meinungsfreiheit gefeiert. Er selbst veröffentlichte mehrere Entschuldigungsvideos zum Vorfall und produzierte in der Folge Beiträge, die den Nazi-Vorwurf quasi-affirmativ $<$ durcharbeiten $>{ }^{13}$ Diese unmittelbaren Reaktionen zum Video sind für die hier verfolgte Lektüre weniger interessant als der Gefühlskern des $<$ Bubenstreichs , den Kjellberg im Finale des Videos darbietet.

Der kommunizierte Affekt ist komplex und gehört in den Bereich der ambivalenten und gemischten Gefühle, welche Sianne Ngai ${ }^{14}$ überzeugend als Mediationen zwischen ästhetischer Erfahrung und politischem Diskurs herausgearbeitet hat: Sprachlosigkeit ist vermengt mit Scham; Erstaunen über das Ergebnis vermischt mit diebischer Freude am gelungenen Konventionsbruch by colonial proxy.

Eine Transaktion innerhalb der neokolonialen Gefüge des Globalkapitalismus - ein weißer Medienprotagonist der ersten Welt beauftragt namenlose Akteure of Color der dritten Welt mit der Aussprache von antisemitischen Parolen - ruft einen komplexen Affekt hervor, der vor allem eine Einfühlung der Zuschauer_innen in die Position des privilegierten Diskurskontrolleurs erreicht. Diese Position ist rassifiziert, denn sie situiert PewDiePie als den Akteur, der über den affektiven Gehalt der transgressiven Sprachhandlung zu entscheiden vermag. Im Rückgriff auf Ngais Konzept der animatedness (Belebtheit) lässt sich der racial discourse entschlüsseln: Mit animatedness beschreibt sie einen Mangel an unabhängigem Handlungswillen, der rassisierten Subjekten in kolonialen und neokolonialen Settings zugeschrieben wird. «[T]his perceived lack of agential vitality renders [racialized] figures not inert but <mechanical> [...]. This fantasy of the racialized body [...] suits an industrial economy in which bodies of color are set into motion like the commodities they produce, and their individual feeling serves only as unmarketizable excess.» ${ }^{15} \mathrm{Kjellbergs}$ antisemitische Bauchrednernummer inszeniert racial bodies als mechanische Puppen, deren funny antics selbst unauthentisch sind und - wichtiger - auf Kommando des weißen und männlichen Diskurskontrolleurs ausgeführt werden. Der reaction shot setzt die weiße Aufnahme rassisierten Gehorsams ins Bild und diktiert den richtigen Affekt fürs Publikum: Scham, Schock, Freude und ironische Moderation der diskursiven Übertretung - kurz, eine Vermischung von Affekten der Schuld und Unschuld, der Nähe und Distanz, die sich mit der inszenierten Entdeckung der eigenen Diskursmacht verbinden. Es geht also um Kjellbergs Inszenierung der eigenen whiteness, gedacht als Machtposition innerhalb eines strukturell rassistischen Gefüges.

Dass Kjellberg beim betreffenden Video die Konsequenzen nicht voll im Blick hatte, kann angenommen werden, auch wenn solches Unwissen immer ein Teil der Performance ist: Seine im Schockmoment ausgestellte Naivität suggeriert Unschuld und authentisches Gefühl - wichtigstes Kapital des YouTube-Vloggers als Affektarbeiter in der virtuellen Aufmerksamkeitsökonomie. ${ }^{16}$ Dieses und die anschließenden Reaktionsvideos ${ }^{17}$ loten die emotionalen
13 Vgl. PewDiePie: The Hitler Simulator (ad), dort datiert 10.3.2017, youtube.com/watch?v=E5qKgw8tDOI, gesehen am 10.7.2018.

14 Vgl. Sianne Ngai: Ugly Feelings, Harvard, London 2007.

15 Kyla Schuller: The Biopolitics of Feeling. Race, Sex, and Science in the 19th Century, Durham 2018, 14.

$16 \mathrm{Hardt} /$ Negri sowie Paolo Virno haben das Konzept der «immateriellen» oder «affektiven Arbeit» für spätkapitalistische Gesellschaften geprägt. In diesen wird nicht nur eine Dienstleistung verkauft, sondern auch ein dazugehöriges Gefühl, ein affektiver Mehrwert des Produkts. Todd Reeser argumentiert, dass neben der Wohlfühlökonomie eine weitere entstanden ist, die vor allem Gefühle des discomfort und der awkwardness als Dienstleistungen produzieren. Vgl. Todd Reeser: Producing Awkwardness. Affective Labor and Masculinity in Popular Culture, in: Mosaic: an interdisciplinary critical journal, Vol. 50, Nr. 4, 2017, 51-68.

17 Vgl. PewDiePie: I'm banned, dort datiert 22.1.2017, youtube. com $/$ watch $? v=61686 \mathrm{cq} 6 \mathrm{~s} 7 \mathrm{c} \&$-frags $=\mathrm{p} \%$ 2Cwn, gesehen am 28.6.2018. 
Spielräume des prankster aus, dessen wirtschaftliches Modell darin besteht, im virtuellen Raum Situationen herzustellen, auf deren Existenz dann mit excessive emoting (Überraschung, Scham usw.) geantwortet werden kann. Diese Emotionsprodukte werden veröffentlicht und monetarisiert. YouTube-Vlogger sind in erster Linie Gefühlsarbeiter_innen, die aus virtuellen Automatismen und Datenströmen Fühlbares herstellen und verkaufen. Affekt ist das Produkt dieses Industriezweiges.

\section{«It's okay to be white»}

In der Nacht zum I. November 2017 tauchten an mehreren US-amerikanischen und kanadischen Universitäten (u.a. Ohio, Moorhead, New Orleans, Silver Spring, Cambridge, Harvard, Alberta) und an anderen Orten minimalistisch gestaltete Zettel auf, die in schwarzer Schrift verkündeten: «It's okay to be white». Die Poster gingen auf eine im Internetforum 4chan lancierte Aktion zurück, in der ein_e anonyme_r Nutzer_in am 31. Oktober 2017 folgenden Text gepostet hatte:

Game Plan: I. anons organize here and elsewhere, print out uniform posters; 2. put on silly halloween costume for anonymity; 3 . posters go up on campuses (and elsewhere) across the world on halloween night; 4 . the next morning, the media goes completely berserk; 5 . normies tune in to see what's going on, see the posters saying <it's okay to be white> and the media \& leftists frothing at the mouth; 6. normies realize that leftists \& journalists hate white people, so they turn on them; 7 . credibility of far left campuses and media gets nuked, massive victory for the right in the culture war, many more /ourguys/ spawned overnight; IMPORTANT REMINDERS: The point is to have MAXIMUM CONTRAST between how evil the media portrays these posters, and how clearly benign they are to normies. ${ }^{\mathbf{1 8}}$

Die Posteraktion, hier abgekürzt als IOTBW, zog konträre Reaktionen nach sich, vergleichbar mit PewDiePies Fiverr prank: Örtliche Polizeiagenturen kündigten eine strafrechtliche Untersuchung der Vorfälle an, Student_innengruppen entfernten die Poster, protestierten gegen «rassistische Sprachgewalt» und veröffentlichten Bilder auf Twitter mit dem Hashtag \#HateCrime. Universitätsleitungen, etwa die Dean of Students Marcia Sells von der Harvard

18 Der Originalbeitrag scheint mittlerweile gelöscht. Ein Screenshot findet sich unter: Don: It's Okay to Be White, in: knowyourmeme, dort datiert 2.11.2017, knowyourmeme.com/ memes/its-okay-to-be-white, gesehen am 28.6.2018.

19 Zit. n. Thomas Williams: Harvard Law Dean Denounces ‘Okay to Be Whites Stickers on Campus, in: breitbart.com, dort datiert 6.11.2017, breitbart.com/big-govern ment/2017/11/06/harvard-law-deandenounces-okay-to-be-white-stickers-oncampus, gesehen am 10.7.2018.

\section{Law School, richteten Presseerklärungen an die Öffentlichkeit:}

[The stickers] were intended to divide us from one another. HLS will not let that happen here. We live, work, teach, and learn together in a community that is stronger, better, and deeper because of our diversity and because we encourage open, respectful, and constructive discourse. ${ }^{19}$

Alle landesweiten Fernsehsender berichteten über die Vorfälle und versuchten mehrheitlich, die Aktion mit der rechtsextremen Alt-Right-Szene in Verbindung zu bringen. Die Ausnahme bildete Fox News, deren Kommentator Tucker Carlson eine wohlmeinende Interpretation des Slogans verteidigte und die 
antirassistischen und distanzierenden Kommentare seiner Kolleg_innen als «anti-white racism» angriff. ${ }^{20}$ Die deutsch-österreichische identitäre Rechte nahm die Aktion positiv auf und schrieb sie Mike Enoch zu, einem bekannten rechtsextremen Aktivisten, der u.a. den Podcast The Daily Shoab produziert. ${ }^{21}$

Was bei PewDiePies prank als spontan-naives Entdecken der eigenen Diskursmacht inszeniert wird, materialisiert sich bei der Aktion als taktisch lancierte Manipulation von Diskursräumen durch eine Diskursguerilla von rechts. IOTBW ist ein prank, der einem Klingelstreich ähnlich die Routinen politisch empfindlicher Öffentlichkeiten - Universitäten und Mainstream-Medien - auslösen und gegen die Sensibilitäten vermeintlicher weißer Normalbürger_innen ausspielen möchte. Die Fabrikation von Empörung und Kritik wird provoziert, um eine Selbstdenunziation der politischen Gegner hervorzurufen, die sich durch einen Kritikreflex und Gefühlswallungen gegen einen vermeintlich <harmlosen〉 Satz ins diskursive Abseits manövrieren sollen. Das postuliert zumindest der game plan. Vorausgesetzte_r Leser_in des Statements und der Reaktionenkaskade ist the average white American, der_die sich in der Folge seiner_ihrer Normalität und okayness beraubt ${ }^{22}$ und von jenen ausgegrenzt fühlen soll, die sagen: «it's not okay to say <it's okay to be white»».

Wie schon bei PewDiePies antisemitischer Aktion werde ich hier nicht den 〈Diskurswert〉 des Statements «It's okay to be white» in den zeitgenössischen USA erläutern. Die relative Aussagelosigkeit des Satzes ist sein Hauptmerkmal. Wie mehrere Kommentator_innen allerdings betont haben, wurden die Poster u. a. neben Gedenkstätten für die Opfer rassistischer Gewalt in den USA platziert oder in direkter Nähe zu universitären Vertretungen von Studierenden of Color und antirassistischen Initiativen. Sie waren also tangential zum semantischen Gehalt Akte einer performativ-rassistischen Gewalt.

Wichtiger als die inhaltliche Auseinandersetzung mit der Phrase ist ein Abschreiten der affektiven Einbettung des pranks. Neben den erwartbaren (und in der Tat erwarteten) Gesten der Empörung, der Kritik und auch des Abwiegelns interessiert hier vor allem, wie die 〈Diskurskontrolleur_innen〉 der diversen beteiligten Messageboards und Internetforen affektiv reagiert haben. Der gefühlskommunizierende reaction shot zu dieser Aktion ist daher nicht in den kritischen Berichterstattungen oder Solidaritätsbekundungen zu suchen, die sich anschlossen.

Die Hauptemotionen, welche die Unterstützer_innen des pranks zur Schau stellten, waren Abgeklärtheit, Coolness und Überlegenheit: Ein Plan hatte funktioniert, jemand war auf den Klingelstreich am Haus der Political Correctness hereingefallen, ein Sieg wurde errungen. Im Reddit-Forum /nostupidquestions/ wurde die Aktion ausgiebig diskutiert («Is the It's okay to be white campaign racist?») und z. B. von Nutzer Kerath am 2. November 2017 wie folgt kommentiert:

It's a simple sentence that was meant to start a game. A game that the only winning move is not to play. And yet, they played it anyway, proving to everyone that it's indeed NOT okay to be white. Master's play from $/ \mathrm{pol},{ }^{23}$ the game was won before
20 Vgl. N. N.: Tucker Carlson defends 4chan's sit's okay to be white campaign, in: Mediamatters for America, dort datiert 3.11.2017, mediamatters.org/video/2017/11/03/ tucker-carlson-defends-4chans-its-okaybe-white-campaign/218454, gesehen am 28.6.2018.

21 Vgl. Johannes Poensgen: It's Okay to be White, in: Blaue Narzisse, dort datiert 14.11.2017, blauenarzisse. delits-okay-to-be-white, gesehen am 28.6.2018.

22 Vgl. Lauren Berlant zu Donald Trump: «Here is the thesis of this piece, which is about the contemporary United States. People would like to feel free. They would like the world to have a generous cushion for all their aggression and inclination. They would like there to be a general plane of okayness governing social relations. It is hard for some to see that the senerous cushion for aggression might conflict with the 'general plane of okayness.m Vgl. Lauren Berlant: Trump or Political Emotions, in: Supervalent Thought, dort datiert 4.8.2016, supervalentthought.com/2016/08/04/ trump-or-political-emotions, gesehen am 28.6.2018.

23 Das Kürzel «/pol» bezieht sich auf das Internetforum reddit. com/r/pol/, das als Sammelpunkt für viele neurechte Tendenzen und Akteure gilt. 
it started. The only thing alt-left is going to achieve now is more people standing against their twisted ideals. ${ }^{24}$

Nutzer hong2hong schrieb:

The left have no way to win this game. If they attack, /pol win because it show that they are racist. If they keep silent, /pol win, because it show to people that its okay to be white. Dont hate your white self/group/people. Whatever the left choose, /pol get the last laugh. $4 \mathrm{D}$ chess everyone. ${ }^{25}$

Wer schon einmal critical race theory oder verwandte Theoriekomplexe unterrichtet hat, wird sich erinnert fühlen: Es gibt fast immer diesen einen männlichen Studenten, der sich spitzfindig eine Provokation innerhalb der Diskussionsparameter ausgedacht hat und diese solipsistisch erläutert. Mittels möglichst viel high theory wird ein transgressives Argument errichtet, das die <impliziten Tabus> eines Seminars entschleiern soll. Übersicht, Kontrolle und Manipulation von Theorie und Debatte zeitigen ein Belohnungsgefühl - man hat ein <meisterhaftes Spiel> gemacht. Angesichts solcher Selbstverliebtheiten wird unser theoretisches Instrumentarium oftmals stumpf - die Diskussion lohnt nicht, weil das Argument <leer> ist, <ausgedacht>. Wichtig ist, so findet man als Lehrende_r schnell heraus, nur der affektive Gewinn des Provokateurs, erfolgreich polarisiert und den Diskurs manipuliert - gespielt - zu haben. Jede mögliche Reaktion bedient diesen Affekt.

\section{Red-Pill-Momente und whiteness}

IOTBW thematisiert Ngais oben genanntes Konzept der animatedness auf andere Weise: Der Mangel an agential vitality (Fremdsteuerung) wird hier für <die Öffentlichkeit> selbst konstruiert. Die Aktion evoziert die Reaktions- und Sprechgewohnheiten westlicher Öffentlichkeiten als leere Routinen, unecht, manipulierbar, mithin fremdsteuerbar. Wie der Klingelstreich die Automatismen der bürgerlichen Welt ausnutzt, um Verwirrung dieser Routinen zu erzie-

24 Aconserva3: Is the It's Okay to be White Campaign Racist?, in: Reddit.com, dort datiert 2.11.2017, reddit.com/r/NoStupidQuestions| comments/7aad2d/is_the_its_okay_to_ be_white_campaign_racist, gesehen am 28.6.2018.

25 Ebd. « 4 DChess) ist ein Internetkürzel für eine Ideologie, nach der die Trolle und Betamännchen des Internets einer höheren Logik und Intelligenz folgen als sogenannte normies oder sheep. Vgl. Don: Trump is playing 4D Chess, in: knowyourmeme, dort datiert 20.7.2017, knowyourmeme.com/memes/ trump-is-playing-4d-chess, gesehen am 28.6.2018. len, so ist die rassistische Transgression darauf ausgerichtet, die Aufregungspunkte medialer Öffentlichkeiten anzureizen. Die öffentliche Meinung wird als Kulisse und Automatismus benutzt, um durch Transgression Empörungswellen zu fabrizieren, gegen die man sich dann affektiv positionieren kann. <The liberal left $>$ wird hier als ebenso mechanisch, manipulierbar und unfunny vorgestellt wie die bodies of color im vorherigen Beispiel.

IOTBW sollte einen Präzedenzfall produzieren, der den vermeintlichen Totalitarismus und Bias der Öffentlichkeit (gleichgesetzt mit <the left〉, <the media>, $<$ political correctness $>$ ) bewiesen werden kann. Da es immer eine Reaktion geben wird, sehen sich die Manipulateur_innen in ihrer Überlegenheit immer bedient. Und da sie sich immer überlegen fühlen können, ist die Faktizität einer <mechanisierten>, verblendeten und damit <unterlegenen> Öffentlichkeit immer schon 

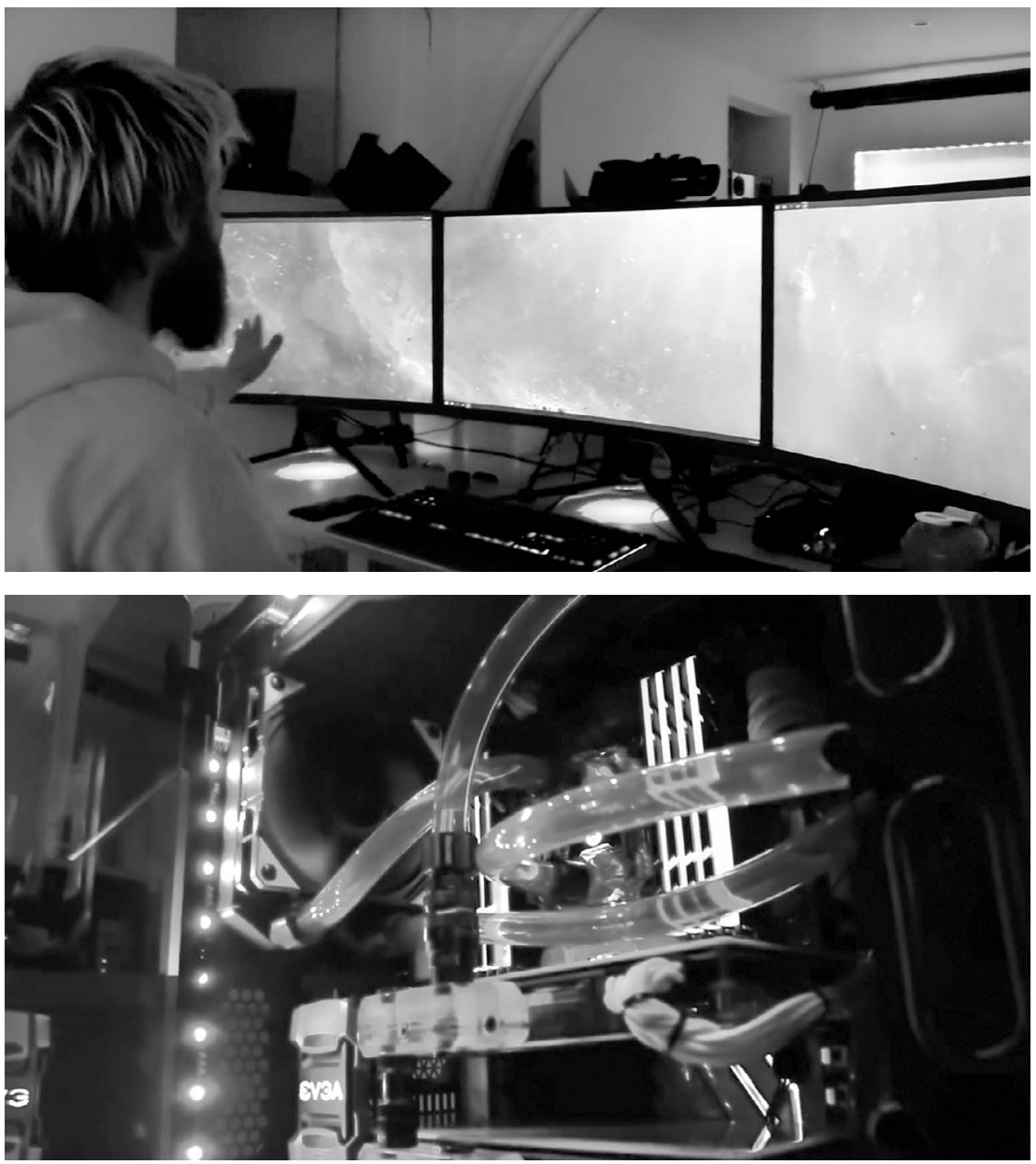

PewDiePie in seinem Studio-Setup (2018) 
bewiesen. Ob Zirkelschluss oder Diskursmanipulation, 4chan- und Reddit-Sympathisant_innen fühlen sich doppelt ins Recht gesetzt. Die Alt-Right bezeichnet solche «Realisierungseffekte ${ }^{26}$ und Beweisschwellen als Red-Pill-Momente: Situationen, in denen ein Mensch den linken Verblendungszusammenhang/die marxistisch-feministische Weltverschwörung> erkennt und zu den zentralen Ideen der neuen Rechten (Ethnonationalismus, Antifeminismus, Xenophobie, Demokratieskepsis usw.) gewissermaßen konvertiert wird.

Im Anschluss stellt sich die Frage, welchen Status whiteness in beiden Beispielen hat - implizit-performativ bei PewDiePie, explizit bei \#IOTBW. Diese Frage berührt den Aspekt, inwiefern solche Phänomene, die generell als Internet Trolling ${ }^{27}$ gefasst werden, Bestandteile eines neurechten, rassistischen und ethnochauvinistischen Alt-Right-Diskurses sind. Mit <wbite $>$ rufen beide pranks eine Diskursposition auf, die als <faktisch prekarisierte> vorgestellt werden soll. Der nicht nur akademischen Problematisierung von whiteness als rassistisch legitimierter Machtposition und white supremacy als ausschließender Strukturhegemonie wird hier ein affektiver Kern des being white auf- oder entgegengesetzt. Die pranks beschwören Weiß-Sein als gesellschaftlich bedrohte, diskriminierte Subjektposition. Dieser Entwurf spielt mehr im Register 〈Klasse〉 als in jenem der 〈Ethnie $\rangle$ : $\langle$ White $\rangle$ wird artikuliert als jene Position, die gesellschaftlich primär durch Drangsalierung, Zensur und eine verweigerte okayness bestimmt ist. $\langle$ White $>$ sei die Klasse der im öffentlichen (verblendeten) Diskurs Verfolgten und Benachteiligten.

Mit dieser affektiven Konstruktion von whiteness wird ein identitätspolitisches Sprechen als <white person> ermöglicht, das dem Vorwurf des Rassismus ausweichen kann, weil es weniger um $<$ Rassenbewusstsein $>$ als um $<$ Klassengefühle $>$ geht. Das ist die Ummünzung der kritischen Kategorie whiteness zur affektiven Identifizierung als white - Basis neurechter und ethnonationalistischer Agitation. Sie läuft als Gewinnstrategie bei beiden pranks mit, angesiedelt auf verschiedenen Ebenen und amplifiziert von spezifischen medialen und gesellschaftlichen Gegebenheiten. Letztlich handeln beide pranks mit einem weißen Subjekt, das zugleich bedroht und diskurskontrollierend, metapolitisch und hochverletzbar aufgestellt ist. Dieses phantasmatische weiße Subjekt ist Surplus des Vabanquespiels

26 Im Sinne eines (Real-Werdens): Die Zirkulation von diskursiven Manövern zeitigt eine reale Situation und bringt ebenjene Öffentlichkeit hervor, in die sie eingreifen wollen. Vgl. Michael Warner: Publics and Counterpublics, in: Public Culture, Vol. 14, Nr. 1, 2002, 49-90.

27 Phillips, Milner: The Ambivalent Internet. Vgl. auch Angela Nagle: Kill All Normies: Online Culture Wars from 4 Chan and Tumblr to Trump and the Alt-Right, Winchester, Washington 2016, $106 \mathrm{ff}$.

28 Phillips, Milner: The Ambivalent Internet, 37, Herv. SStr. der Diskursguerilla. Mit diesem Ergebnis kann u.a. eine der Grundannahmen zum Phänomen des Trolling kritisiert werden, derzufolge Online-Trolle gesellschaftliche Antagonismen aufgreifen, verschärfen und transgressiv zuspitzen und damit gesellschaftliche Wirklichkeiten <reflektieren〉: «These sort of antagonistic memes weaponize existing cultural logics, and thus reflect the antagonisms pervasive in embodied spaces as well.» ${ }^{28}$ Der Befund wäre umzudrehen: Die affektiven Einbettungen rechter Trollingmanöver produzieren und realisieren eben jene rassistischen Verkörperungen und Identitäten als Waffen gesellschaftlicher Spaltung. Ergebnis ist nicht nur eine <virtuelle> Verzerrung des Diskurses, sondern ein identifikationsstiftendes Gefühl der Unterdrückung, das sich an scheinbaren Fakten festmachen kann: eine fühlbare <weiße> Gegenöffentlichkeit. 


\section{Drei Vorschläge: Against Atmosphere}

Ich möchte drei Konsequenzen dieser Lektüren vorschlagen, die kritischen Ansätzen zur Alt-Right helfen sollen.

Erstens: Die Alt-Right ist ein affektives Kontinuum. Rechtsextreme Agitation und das (finanziell äußerst lukrative) rechte <role playing> von Medienprotagonisten wie PewDiePie sollten nicht mehr trennscharf in bate speech und ironische Appropriation - also in 〈Nazis〉 und 〈Ironisierende〉 - sortiert werden. Beide Beispiele nutzen ähnliche Diskursmanipulationen und Affektroutinen, die zentral an der Konstruktion von Situationen arbeiten, in der eine <subalterne> weiße Identität sich gewissermaßen von selbst artikuliert. Aus dieser Sicht sind die Alt-Right, ihre juvenilen Streichkolleg_innen und deren Manöver weniger einem <rechten Gedankengut > bzw. <inhaltsleerem Trolling> verpflichtet, sondern arbeiten gemeinsam an weißer Gefühlsproduktion und der medialen Herstellung rassistisch antagonisierender Atmosphären. Was die Rechte selbst als (ironisches oder kritisches) Ausloten der angeblich bedrohten Meinungsfreiheit propagiert, bewirkt im Effekt eine Verknappung des Sagbaren und der Diskursmöglichkeiten. ${ }^{29}$

Zweitens: Die Alt-Right ist keine Kritik. Die Guerillataktiken der Alt-Right sind für sich selbst uninteressant, weshalb ihnen das Belohnungsmoment der Kritik- oder Kulturleistung zu entziehen ist. Eine ebensolche Belohnung vollzieht z.B. Angela Nagle, die in ihrer vielzitierten Studie Kill All Normies zu rechten Trollen, chauvinistischen Betamännchen und provokativen Nerds diese als Kritikbewegungen und Gegenöffentlichkeiten zu einem 〈linken Konsens〉 beschreibt. Die Rechte biete, so Nagle, die derzeit einzige «exciting, fun and courageous» Alternative zu linken Partikularkritiken und cliquenhafter Identitätspolitik, die sie als «anti-free speech, anti-free thought, anti-intellectual [...] movement $\gg^{\mathbf{3 0}}$ bezeichnet. Solche Reiz-Reaktionsmodelle sind verkürzend und selbst bereits Resultat der Antagonisierungen, die von rechts betrieben werden. Dass die Diskursspiele der Alt-Right für Nagle einen Eros der Transgression und der Kritik beanspruchen können, zeigt vor allem deren atmosphärische Hegemonie an. Solange die neurechte Diskursguerilla als Agentur für <interessante Überschreitungen> von Diskursgrenzen betrachtet wird, an denen die Verfehlungen und Verhärtungen eines (vermeintlich monolithischen) linken Projekts ablesbar werden, tragen wir zur rechten Affekthegemonie bei.

Drittens: Die Alt-Right ist keine $<$ Hass $>$-Bewegung. Ebenso wie Trolling eine ungeeignete, weil entpolitisierende Beschreibung ist, wirkt das generell für rechts vorbehaltene Emotionsetikett verkürzend: 〈Hass $>$ zieht sich, wie Pansy K. Duncan anhand zahlreicher Beispiele ausführt, ${ }^{31}$ als roter Faden durch die Mainstreamverhandlung neofaschistischer Kräfte. Duncan argumentiert, dass
29 Als Beispiel für die Realienproduktion dieser Diskurse lässt sich die im Mai 2018 in London organisierte Großdemonstration ‘Day for Freedom anführen, auf der zahlreiche Meinungsführer der Alt-Right zu mehreren tausend Menschen gegen Migrationspolitik und weiß-britischen Kulturverlust sprachen - ein wichtiges Vernetzungstreffen der neuen Rechten aus USA und Europa. Anlass des Protests war die Verurteilung des YouTube-Pranksters Count Dankula zu einer Geldbuße, der seinem Mops beigebracht hatte, die Pfote zum Hitlergruss zu heben. Vgl. N. N.: London Holds ‘Day of Freedom Protest Aimed to Protect Free Speech, in: Sputniknews, dort datiert 6.5.2018, sptnkne.ws/hzM5, gesehen am 28.6.2018.

30 Nagle: Kill All Normies, 113. 31 Vgl. Pansy Kathleen Duncan: The Uses of Hate. On Hate as a Political Category, in: M/C Journal, Vol. 20, Nr. 1, 2017, o. S. 
diese Zuweisung von <Hass> als Charakteristikum der Alt-Right aber vor allem ein «rhetorical projectile» zur bequemen Komplexitätsreduktion und Distanzierung ist: «a constellation from which hate emerges as, a) inherently problematic, b) localizable to the <alt-right>, and, c) the primary engine of the various activities and expressions we associate with them.» ${ }^{32}$ Duncans Plädoyer gegen die Verkürzung der neuen Rechten auf eine singuläre Emotion und für eine komplexe Analyse der «economic, social, political and affective forces that energize it» schließe ich mich an: Die besprochenen Beispiele zeigen nicht nur auf, wie wenig die Aktionen und Interventionen der Alt-Right auf $<$ Hass $>$ als auslösende Emotion reduziert werden können, sondern weiter, wie banal, ausgedacht, juvenil und zugleich überlegt, marktorientiert, technikaffin und affektzentriert der Diskurs um Antifeminismus, Ethnonationalismus, white racial pride und Xenophobie auftritt. Die neue Rechte oder Alt-Right ist mithin <casual>, d. h. affektiv, atmosphärisch, intim und - innerhalb der durch Klicks, view counts und andere Quantifizierungsmechanismen börsenähnlichen digitalen Öffentlichkeit - so effektiv wie lukrativ. Wie der Exkurs zu whiteness in beiden Beispielen außerdem zeigt, bedient sich die neue Rechte adaptiv und kolonisierend an klassisch linken Instrumentarien wie der Identitätspolitik, mit affektiven, und d.h. realen, Folgen. Eine Konsequenz also ist, dass die notwendige, kritische Gegenbewegung zum Kulturkampf von rechts ihre eigenen Codes unterbrechen sollte - Duncan spricht hier von «critical code-breaking»-, um den hegemonialen Affekten der Alt-Right entgegenzutreten.

An diese drei Konsequenzen möchte ich eine kurze, methodische Überlegung anschließen. Hauptziel des Vergleichs war nicht, populäre YouTuber wie PewDiePie dem Rechtsextremismus zuzuschlagen. Ich sehe darin jedoch kein kategoriales Problem, denn beide Kanäle arbeiten auf dieselben Effekte hin. Der Punkt ist dabei, dass eine Analyse der Online-Rechten auch auf diese Effekte abstellen muss und nicht nur auf die Frage, wer $<$ rechts denkt $>$. Die Forschung zu neurechten Bewegungen in den sozialen Medien und anderswo wird weitgehend mit veralteten Parametern der Personalpolitik geführt: Es geht um Köpfe und Figuren, die gefährliches Gedankengut und bate speech verbreiten. Einschlägige Veröffentlichungen zum Thema Alt-Right unternehmen daher oft eine Inventarisierung der Akteur_innen. ${ }^{33}$ Implizit in diesem Modell ist eine Vorstellung von rechtsgerichteten Menschen als lokalisierbare Träger_innen von <infektiösen> Ideen und Denkstrukturen, die sich epidemisch verbreiten. Diese personelle bzw. epidemologische Methodik ist angesichts der hier disku-

32 Duncen: The Uses of Hate. 33 Vgl. die «hate map» des Southern Poverty Law Center (splcenter.org/hate-map) oder die vom antifaschistischen pressearchiv und bildungszentrum berlin e.v. (apabiz) betriebene Website rechtesland.de, beides gesehen am 10.7.2018. tierten Netzwerkeffekte des Internetzeitalters schwierig geworden.

Dem epidemologischen Modell muss eine andere Analyserichtung hinzugefügt werden, die sich um interpersonelle, strukturelle, momentane und identifikationsstiftende Affekte kümmert. Die derzeitige Alt-Right-Bewegung besteht weniger aus Ideolog_innen als Gefühlsarbeiter_innen: Sie schaffen informelle und provisorische Affektgruppen, bündeln punktuell Aufmerksamkeiten und 
realisieren Antagonismen. Sie schaffen also Atmosphären, die im Flächeneffekt das vollziehen, was die Vordenker_innen dieser postmodernen Rechten als <Metapolitik> bezeichnet haben: «die Besetzung von Feldern im vorpolitischen Raum». ${ }^{34}$ Intention und Effekt der Alt-Right-Strategien ist also ein diskursiver Klimawandel im Wortsinn - Verknappung und Extremisierung des Sagbaren, Intensivierung der Gefühlsräume, schnelle Wechsel von heißen (Wut) und kalten (Übersicht, Coolness) Affekten. Die rassistische Rechte provoziert extremtemperierte Debatten, sie profitiert von strategisch geplanten Diskursbeben und Flutwellen der Empörung. ${ }^{35} \mathrm{Ihr}$ Ziel ist ein apokalyptisches und tödliches Diskursklima.

Wir sind also nicht primär Epidemiolog_innen, die eindämmen und kurieren, um das $\langle$ Normale $>$ (Wir) und das $\langle$ Pathologische $>$ (die Rechten) klar voneinander geschieden zu halten. ${ }^{36}$ Die kritische Gegenbewegung zur neuen Rechten sollte sich einer <Klimaforschung > verschreiben, welche die atmosphärischen Extremismen in unseren media environments beschreibt und nach Lösungen sucht. Wir alle sind den Folgen des diskursiven Klimawandels ausgesetzt, auch wenn sie nicht für alle gleich tödlich sind: Der Klimawandel fordert Opfer along racial lines ${ }^{37}$ womit Rassismus im Anthropozän zur menschgemachten Naturgewalt aufgestiegen ist. Wir müssen nach Wegen suchen, dem klimatischen und atmosphärischen Rassismus in unseren medialisierten Öffentlichkeiten etwas entgegenzusetzen. Einzelne Wettermacher zu denunzieren oder argumentativ kaltzustellen, ändert am Klimawandel wenig. A hard rain's a-gonna fall.
34 Karlheinz Weißmann, zit. n. Andreas Speit: Der Oberintellektuelle, in: taz, 21.4.2018, www.taz.de| !5399096l, gesehen am 28.6.2018. Vgl. auch: Massimiliano Capra Casadio: The New Right and Metapolitics in France and Italy, in: Journal for the Study of Radicalism, Vol. 8, Nr. 1, 2014, 45-86.

35 Vgl. Sylvia Hurtado: The Campus Racial Climate: Contexts of Conflict, in: The Journal of Higher Education, Vol. 63, Nr. 5, 1992, 539-569.

$36 \mathrm{Vgl}$. Sander Gilman, James Thomas: Are Racists Crazy? How Prejudice, Racism, and Antisemitism Became Markers of Insanity, New York 2016.

$37 \mathrm{Vgl}$. W. A. Baldwin: Resilience and race, or climate change and the uninsurable migrant. Towards an anthroporacial reading of (race), in: Resilience, Vol. 5, Nr. 2, 129-143. 